\title{
On the ephemeris of the eclipsing polar HU Aquarii
}

\author{
A.D. Schwope ${ }^{1, \star}$ and B.D. Thinius ${ }^{2}$ \\ 1 Leibniz-Institut für Astrophysik Potsdam (AIP), An der Sternwarte 16, 14482 Potsdam, Germany \\ 2 Inastars Observatory, Hermann-Struve-Str. 10, 14469 Potsdam, Germany
}

The dates of receipt and acceptance should be inserted later

Key words stars: individual: HU Aqr - binaries: eclipsing - stars: cataclysmic variables

The magnetic cataclysmic variable HU Aquarii displayed pronounced quasi-periodic modulations of its eclipse timing. These were interpreted in terms of the light-travel time (LTT) effect caused by a circumbinary planet or planetary system. We report new photometric observations that revealed another precise eclipse timing for the October 2013 epoch, the first obtained in a high accretion state after many years in low or intermediate states. The eclipse was observed to occur earlier by $95.3 \pm 2.0 \mathrm{~s}$ or $62.8 \pm 2.0 \mathrm{~s}$ than expected for an assumed linear or quadratic ephemeris, respectively. The implied apparent strong evolution of the orbital period calls for a revision of the current planetary model or the planetary parameters. The object deserves further monitoring to uncover the true nature of the observed variability and to constrain the properties of the proposed planet or planetary system. The new observations prove that advanced amateur equipment can successfully be used in the growing field of planet search in wide circumbinary orbits via the LTT effect.

(C) 2014 WILEY-VCH Verlag GmbH \& Co. KGaA, Weinheim

\section{Introduction}

HU Aqr is an eclipsing magnetic cataclysmic variable with a $125.0 \mathrm{~min}$ orbital period. When discovered in 1993 as the optical counterpart to the soft X-ray and EUV sources RX J2107.9-0518/RE2107-05 (Hakala et al. 1993; Schwope et al. 1993) it was the brightest eclipsing object displaying the most extended eclipse. Those properties triggered broad observational studies to disentangle accretion phenomena and the accretion geometry in a strongly magnetic environment. Particular emphasis was given to model the detailed eclipse structure.

Comprehensive X-ray and EUV observations with the ROSAT and EUVE satellites took place between 1992 and 1998 (Schwope et al. 2001). These studies established the eclipse egress as fiducial mark to determine the orbital period and a long-term ephemeris. The ingress into eclipse was found to be strongly affected by absorbing matter in the accretion curtain preventing an unequivocal determination of eclipse centre. The 31 epochs given for the eclipse egress that were based on soft X-ray observations already displayed systematic offsets with respect to a linear ephemeris of $\pm 5 \mathrm{~s}$. The size of the observed effect was still compatible with a migration of the accretion spot over the surface of the white dwarf.

Monitoring observations with high-time resolution were continued at X-ray and optical wavelengths in the new millennium. Schwarz et al. (2009) presented a further set of 62 eclipse epochs and were the first to discuss the timing residuals, that were then larger than the size of the white dwarf,

\footnotetext{
* Corresponding author: e-mail: aschwope@aip.de
}

in terms of an unseen third body and derived a possible mass of $M_{3} \gtrsim 5 M_{\mathrm{Jup}}$ for a planetary companion.

Oian et al. (2011) added a further 11 eclipse epochs and claimed the discovery of a circumbinary planetary system around the accreting binary. This hypothesis is debated. Their data seem to be offset from a more comprehensive data set presented by Goździewski et al. (2012) and the proposed planetary system seems to loose stability on relatively short time scales (Horner et al. 2011; Wittenmyer et al. 2012). The study by Hinse et al. (2012) on the other hand supports at least a two-planet scenario. Opportunities to reconstruct the evolution of the close binary through the presence of a planetary system were studied by Portegies Zwart (2013). The stellar and binary parameters were updated by Schwope et al. (2011) who derive a total mass of the close binary of $0.98 \pm 0.10 \mathrm{M}_{\odot}$.

The frequent low- and intermediate accretion states of the object prevented further detailed X-ray observations during the past 12 years. In July 2013 a high accretion state was noticed by monitoring observations with the robotic telescope STELLA at Tenerife. Swift and XMM-Newton observations were triggered to study the $\mathrm{X}$-ray emission in a high accretion state. The observations with XMMNewton were scheduled immediately after re-appearance of the source in the visibility zone of XMM-Newton and took place eventually on October 25.

At that occasion attempts were made to obtain simultaneous ground-based data (Schwope et al. 2014, in preparation). Here we report quasi-simultaneous optical observations that were obtained utilizing the private equipment of the Inastars observatory Potsdam (IOP, B15, N 52.42392, E 13.012892). 


\section{Observations and data reduction}

HU Aqr was observed with a 14 inch Celestron reflector of Schmidt-Cassegrain type during six clear nights in October 2013. The telescope is permanently installed on the roof of a one-family dwelling in a suburb of Potsdam, the capital of the state of Brandenburg (Germany). An Optec NextGEN WideField 0.5X telecompressor revealed an aperture ratio of $\mathrm{f} / 5.5$ and a field of view of $24 \times 16$ minutes of arc. The data were obtained through an ASTRONOMIK1 filter which blocks typical emission lines at light-polluted sites and has almost full transparency in two windows that range from $450-520 \mathrm{~nm}$ and $640-690 \mathrm{~nm}$, respectively. The camera used, an SBIG ST-8XME, features a CCD with $1530 \times 1020$ pixels (pixel size $9 \times 9$ microns) and was used mostly with a $2 \times 2$ binning. The temperature of the CCD is actively controlled and was set to $-25.0^{\circ} \mathrm{C}$ during the observations which was achieved with an rms deviation of about $0.3^{\circ} \mathrm{C}$. The telescope/camera system is mainly used for observations of minor planets. Several new discoveries are confirmed by the minor planet center (MPC) of the IAU (see e.g. http://thinius.net/discstatus.htm) Since this was the first time, that the equipment was used for timeresolved photometry with an as high as possible resolution, some experiments were made using different CCD binning and exposure time per frame to find a compromise between highest possible time resolution and an acceptable signalto-noise ratio.

A summary of the observations reported in this paper is given in Table 1, which lists the exposure time, the achieved time resolution, the number of frames and the time interval covered during each of the nights. During the last night, beginning October 30, a total of 1995 science frames were obtained. Almost all of those, 1973 frames, were taken with an exposure time of $4 \mathrm{~s}$. During the two eclipses, however, the exposure time was changed to $20 \mathrm{~s}$ ( 7 frames) and $60 \mathrm{~s}$ (15 frames) in an attempt to more securely measure the remaining brightness of the binary. Those modifications of the observing sequence resulted in a corresponding less accurate determination of the eclipse time (see Tab. 2).

The start time of each observation and the exposure length was written into the fits headers generated upon readout. In the context of the current paper the timing accuracy of the data is crucial. The computer equipment was correlated with a time signal of atomic clocks every five minutes via the Network Time Protocol (NTP). The synchronization process runs on the measuring computer with high priority. For time synchronization times servers in Germany, the USA as well as India were used. The list of potential time servers comprises 10 redundant stations. The time difference between time server and PC was logged for each synchronization run. Different package transmission times were reported for the servers located at different continents, the measured time differences between server and PC how-

\footnotetext{
${ }^{1}$ http://www.astronomik.com/de/photographic-filters/cls-ccdfilter.html
}

Table 1 Time-resolved photometric observations of HU Aqr obtained at Inastars Observatory in October 2013. Given are the observation date, time interval covered, exposure time, time resolution, and the number of frames obtained per night.

\begin{tabular}{lcccr}
\hline Date & Interval & $t_{\exp }$ & $\Delta t$ & \# frames \\
& JD +2456588. & {$[\mathrm{s}]$} & {$[\mathrm{s}]$} & \\
\hline 20131022 & $0.29217-0.36269$ & 3.0 & 6.1 & 976 \\
20131023 & $1.31179-1.37931$ & 3.0 & 6.1 & 277 \\
20131024 & $2.24323-2.42821$ & 4.0 & 6.9 & 1903 \\
20131026 & $4.26040-4.38630$ & 8.0 & 10.0 & 677 \\
20131029 & $7.27689-7.39935$ & 2.0 & 3.8 & 1951 \\
20131030 & $8.23877-8.41131$ & 4.0 & 6.9 & 1995 \\
\hline
\end{tabular}

ever were found to be stable with a scatter of about $10 \mathrm{~ms}$ or even better.

The CCD software writes images with 1 second time resolution by cutting the fraction of a second. The times written to the CCD headers are thus biased by $-0.5 \pm 0.5 \mathrm{~s}$. Shutter latency measurements gave a mean latency of $0.77 \pm$ $0.02 \mathrm{~s}$. Hence, all times measured on the original data were corrected afterwards by $+1.27 \mathrm{~s}$ and a systematic uncertainty of $0.52 \mathrm{~s}$ was added to the estimated statistical uncertainties. Confidence that the systematic uncertainties are not larger than this value is derived from the positions of minor planets that were determined with the same instrumental setup. The positions reported to the IAU-MPC agree with the orbit predictions always better than one arc-second.

The raw CCD frames were bias-corrected, darksubtracted and flat-fielded using calibration data obtained during each of the nights. For this purpose average frames of each 50 sky-flats, bias- and dark-frames taken immediately before the observations with the same instrumental setup and CCD temperature were used. No trend in the bias values was recognized.

Afterwards, differential photometry using ESO-MIDAS routines was performed with respect to comparison star 'C' (USNO B1.0 0846-0616384, 2MASS coordinates $\mathrm{RA}(\mathrm{J} 2000)=21: 07: 59.091, \mathrm{DE}(\mathrm{J} 2000)=-05: 18: 49.62$, 14.696mag, Schwope et al. 1993). Firstly, the centroids of the stellar images of both the target and the comparison star were determined via two-dimensional Gaussian fits on a number of images with good detections of both stars to determine the pixel offset between them. Then aperture photometry was performed on each CCD frame using concentric apertures for object, nomansland, and sky, centered initially on the comparison star, then at the offset position using the same apertures. With this procedure a brightness measurement was possible even during eclipses of HU Aqr, when the source was too faint for centering. Errors of individual measurements were determined taken into account the sky brightness, the object brightness and the read-noise properties of the CCD. The quantity further investigated is the flux ratio between the target and the comparison star, also referred to as relative flux. 


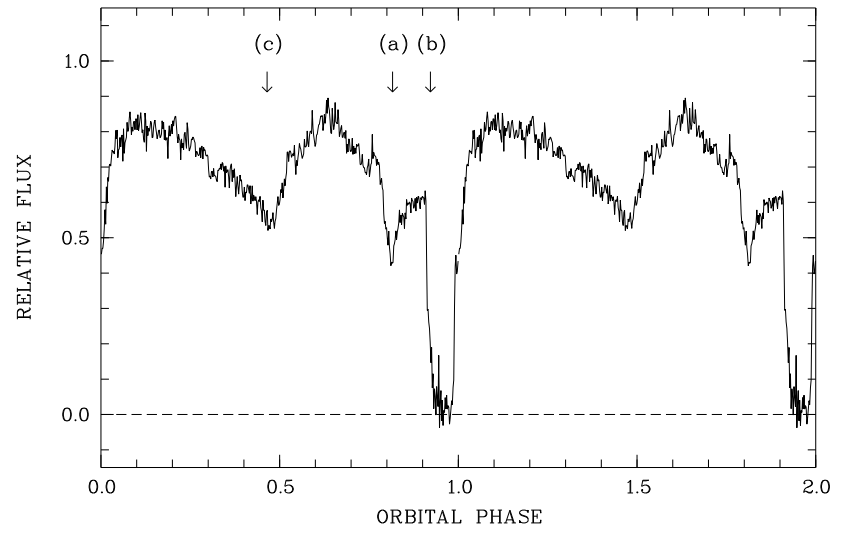

Fig. 1 Phase-averaged light curve of HU Aqr obtained October 2013 at Inastars Observatory (500 phase bins). Orbital phase refers to a linear ephemeris $\mathrm{BJD}(\mathrm{TDB})=$ $2449102.92004+E \times 0.086820400$

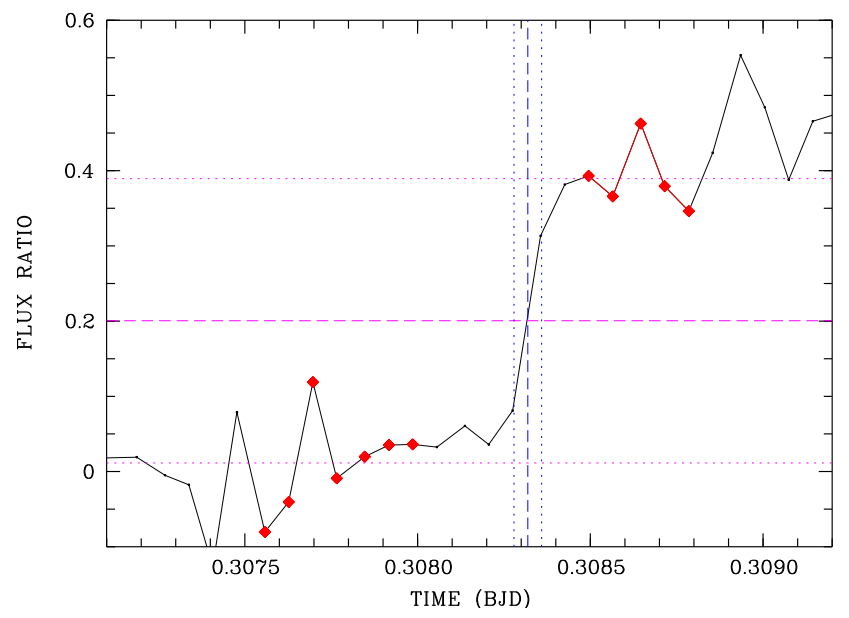

Fig. 2 Determination of eclipse egress on October 24. One data point represents a $4 \mathrm{~s}$ integration, the time resolution is $6.9 \mathrm{~s}$. Shown with dotted vertical lines are mean eclipse and out-of-eclipse values used to compute the halflight value using data shown with larger symbols. The measured time of egress was read at half light and shown with a vertical dashed line. The timing error was set to half a resolution element and is indicated by vertical dotted lines.

\section{Analysis and results}

\subsection{Eclipse parameters}

All timing data were put on the same time scale as previous data. The centers of individual CCD frame times were corrected for arrival at the solar system barycenter and were put on an atomic time scale by adding $32.184 \mathrm{~s}$ for the conversion from TAI to TDT, and $35 \mathrm{~s}$, the current amount of leap seconds.

Initially, the linear part of the two-planet LTT fit by Goździewski et al. (2012), BJD(TDB) = $2449102.92004+E \times 0.086820400$, was used to convert times to binary phase. All 7779 data points were

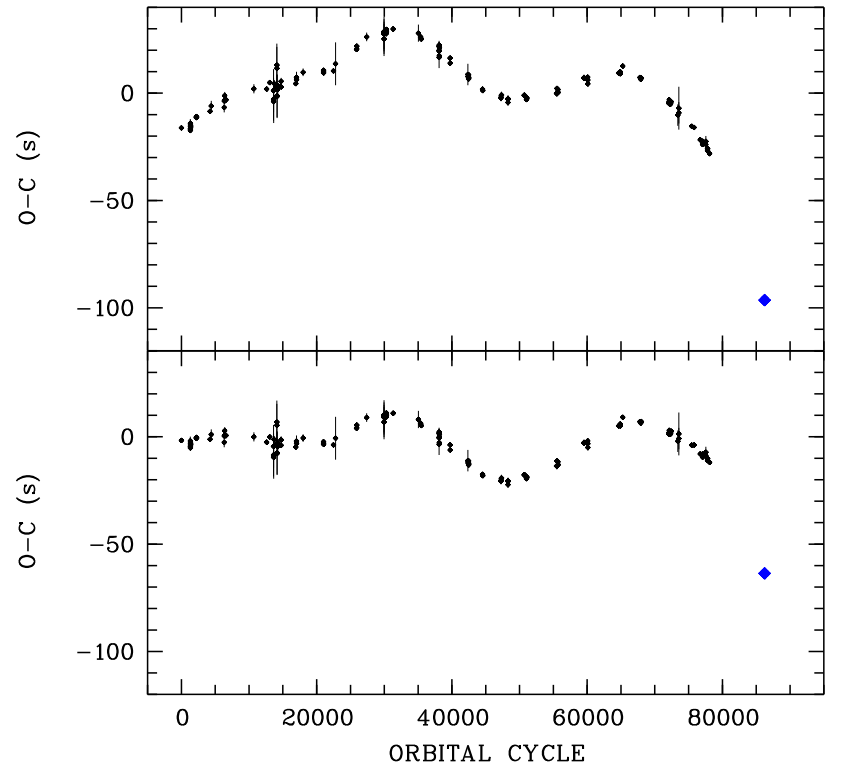

Fig. 3 Observed minus calculated times of eclipse egress of HU Aqr. Data given in Goździewski et al. (2012); Schwarz et al. (2009); Schwope et al. (2001) are shown with small circles, the new result obtained here is shown with a rhomb. In the upper panel the residuals are shown with respect to a linear ephemeris, in the lower panel with respect to a quadratic ephemeris.

phase-folded and binned into 500 phase bins to generate the light curve displayed in Fig. 1. The relative brightness of the object and certain features of the light curve which are also labeled in the figure clearly indicate a high accretion state (for comparison see the collection of light curves obtained in intermediate and high states in Schwope et al. 2001, their Fig. 3). The features are: (a) a pronounced pre-eclipse dip well separated from the eclipse and centered about 0.14 phase units before eclipse centre; (b) a short ingress phase of the accretion stream/curtain after eclipse of the white dwarf; (c) the absence of a flat-bottom faint phase. Both the centre phase of the pre-eclipse dip, $\Delta \phi=-0.140 \pm 0.002$ and the phase of half intensity during dip ingress, $\Delta \phi=-0.175 \pm 0.002$ (both measured with respect to the centre of the eclipse), are comparable to the highest accretion state reported by Schwope et al. (2001). Those early phases of the pre-eclipse dip indicate a late coupling of the accretion stream onto the magnetic field due to the high ram pressure of the accretion stream.

The times of individual ingress and egress were measured by averaging a few data points before and after ingress/egress, computing the half-light intensity and reading the times with a cursor from a graph of the light curve. Figure 2 illustrates the procedure for data obtained on October 24. Uncertainties of individual measurements were set to half the time resolution achieved, unless a data gap deteriorates the timing accuracy further. All ingress and egress timings corrected for the bias of $1.27 \mathrm{~s}$ are listed in Table 2 
Table 2 Times of eclipse ingress and egress. Times are given in BJD(TDB). Errors given do not include the systematic uncertainty of $0.5 \mathrm{~s}$.

\begin{tabular}{cclrlr}
\hline $\begin{array}{c}\text { Date } \\
\text { (MMDD) }\end{array}$ & Cycle & $\begin{array}{l}t_{\mathrm{i}} \\
+2456588\end{array}$ & $\begin{array}{r}\Delta t_{\mathrm{i}} \\
{[\mathrm{s}]}\end{array}$ & $\begin{array}{l}t_{\mathrm{e}} \\
+2456588\end{array}$ & $\begin{array}{r}\Delta t_{\mathrm{e}} \\
{[\mathrm{s}]}\end{array}$ \\
\hline 1022 & 86217 & 0.307269 & 3.0 & 0.313872 & 3.0 \\
1023 & 86229 & 1.348875 & 15.0 & & \\
1024 & 86240 & 2.303966 & 3.5 & 2.310700 & 3.5 \\
1024 & 86241 & 2.390769 & 3.5 & 2.397546 & 3.5 \\
1026 & 86263 & & & 4.307468 & 8.0 \\
1026 & 86264 & 4.387604 & 5.0 & & \\
1030 & 86309 & 8.294531 & 3.5 & 8.301313 & 5.0 \\
1030 & 86310 & 8.381423 & 8.0 & 8.38839 & 30 \\
\hline
\end{tabular}

The times in the list were converted to binary cycle, the integer part was subtracted and phases of ingress and egress were computed as weighted averages of individual measurements, $\left\langle\phi_{\mathrm{i}}\right\rangle=-0.08462 \pm 0.00023$ and $\left\langle\phi_{\mathrm{e}}\right\rangle=$ $-0.00715 \pm 0.00026$, respectively. The measured eclipse length is $581.1 \pm 3.7 \mathrm{~s}$. This value is consistent with the eclipse length in the 1993 high state, 584.6 s (Schwope et al. 1997), measured with the high-speed photometer MCCP attached to the Calar Alto 2.2m telescope. The MCCP-data were obtained with high time resolution that was sufficient to resolve eclipse ingress and egress.

The mean epoch of eclipse egress determined here is $\mathrm{BJD}(\mathrm{TDB})=2456590.397536 \pm 0.000026$ for cycle 86241 . The average value for the named cycle is different by $1.7 \mathrm{~s}$ from the actually measured value.

\subsection{Eclipse ephemeris}

The newly determined time for the eclipse egress was combined with those reported previously in the literature (Goździewski et al. 2012; Schwarz et al. 2009; Schwope et al. 2001). The data obtained by Qian et al. (2011) were not included because these were shown to be offset from data obtained at similar epochs for an unknown reason (see the discussion in Goździewski et al. 2012). Inclusion of those data does not change our results significantly.

A weighted linear regression to all 162 data points yields the linear ephemeris for the eclipse egress

$$
\begin{aligned}
\operatorname{BJD}(\mathrm{TDB})= & 2449102.9201891(12)+ \\
& E \times 0.086820403874(23)
\end{aligned}
$$

(numbers in parenthesis give formal $1 \sigma$ uncertainties, reduced $\chi_{\nu}^{2}=1583$ for 160 d.o.f.). The residuals with respect to this linear fit are shown in the upper panel of Fig. 3. The latest data point derived in the current paper indicates a very significant apparent decay of the orbital period over the last two years so that the eclipse is early by $95.3 \pm 2.0 \mathrm{~s}$ with respect to the best-fit linear ephemeris and by $67.0 \pm 2.2 \mathrm{~s}$ with respect to the last data point presented by Goździewski et al. (2012). When the data of Oian et al. (2011) are included in the fit, the parameters are the same within the errors, the estimated errors remain unchanged, only the goodness of fit statistics is changed slightly, reduced $\chi_{\nu}^{2}=1490$ for 170 d.o.f..

During the time interval 1993 - 2011 the derivative $|\mathrm{d}(O-C) / \mathrm{d} P|$ remained below $0.7 \times 10^{-7}$, the average value for the last two years was $1.1 \times 10^{-7}$.

A quadratic fit of the form BJD $(\mathrm{TDB})=T_{0}+P E+$ $1 / 2 P \dot{P} E^{2}$ reveals a better but still completely unsatisfactory representation of the data: $T_{0}=2449102.9200230 \pm$ $0.0000014, P=0.08682042449 \pm 0.00000000010$, and $\dot{P}=(-616.1 \pm 2.9) \times 10^{-14}$ (reduced $\chi_{\nu}^{2}=417$ for 159 d.o.f.). The residuals of the quadratic fit are also shown in Fig. 3. The latest data point is early by $62.8 \pm 2.0 \mathrm{~s}$ with respect to the best-fit quadratic ephemeris and by $50.0 \pm 2.2 \mathrm{~s}$ with respect to the last data point presented by Goździewski et al. (2012). We note that the quadratic fit may be regarded just as a parameterization of our ignorance of the underlying behavior of the source and the relevant physical processes. The usual suspectives that could be made responsible for a quadratic term, the mechanism described by Applegate (1992) and magnetic braking through gravitational radiation, seemingly do not provide sufficient angular momentum loss to explain the large observed rates (see e.g. the discussion in Schwarz et al. 2009; Vogel et al. 2008).

The new linear and quadratic ephemerides differ from the last published values by Goździewski et al. (2012). Using our formal $1 \sigma$ uncertainties the differences appear to be real, using the uncertainties derived by Goździewski et al. (2012) only the value of the quadratic term appears to be significantly different. Their implied period derivative, $\dot{P}=705 \pm 14 \mathrm{~s} \mathrm{~s}^{-1}$, from their fitted value $\beta=0.5 P \dot{P}=$ $(3.06 \pm 0.06) \times 10^{-13}$ day cycle $^{-2}$, indicates an even larger period change than our quadratic fit. However, the parameters and uncertainties derived in the two papers were derived for different models, the model by Goździewski et al. (2012) taking the LTT-effect of two planets with degenerate parameters into account. A more meaningful comparison of the parameters would benefit from the application of the same model.

\section{Discussion and conclusion}

The latest addition to the measured set of eclipse timings has revealed a very significant apparent decay of the orbital period. It is the first reported measurement of eclipse egress after two years without new data. We checked the reliability of the new data point by inspecting the data obtained quasi-simultaneously with XMM-Newton and found both data sets in agreement within a few seconds. Any possible mis-match of optical and X-ray timing data is too small to explain the huge trend in the $(O-C)$ data shown in both panels of Fig. 3 The results of the satellite data and a more thorough discussion of the relative timing between $\mathrm{X}$-ray and optical data will be will be published elsewhere (Schwope et al. 2014, in preparation). 
A.D. Schwope \& B.D. Thinius: On the ephemeris of HU Aqr

The new data point lies outside the $(O-C)$ ranges of recent studies (Goździewski et al. 2012; Hinse et al. 2012; Wittenmyer et al. 2012). Hinse et al. (2012) were discussing a two-planet model with a summed amplitude of $23.4 \pm 0.1 \mathrm{~s}$, too small, to make it compliant with our large $(O-C)$ s. Their best-fit model also predicts increasingly later arrivals of the eclipse for cycle numbers $>72000$, contrary to what is observed. Goździewski et al. (2012) were trying to fit the complete dataset with a two-planet model which gave apparently unphysical solutions only. Their linear model revealed massive third bodies, $M_{3} \simeq 10 \mathrm{M}_{\odot}$. This model could probably be trimmed to reflect the large observed $(O-C)$ values, such a solution, however, would be mainly of academic interest. The quadratic model reveals an outer planet with eccentricity close to one, which was regarded unphysical by Goździewski et al. (2012) themselves. A single planet model was found to be viable by ignoring data not obtained with the OPTIMA camera. This model with an amplitude of about $15 \mathrm{~s}$, however, is discarded by our result, which also discards the underlying assumption that the mix of data obtained at different wavelengths makes a meaningful fit so difficult.

The planetary parameters reported in those studies therefore need revision.

The current peak-to-peak amplitudes of the $(O-C)$ curves are $74 \mathrm{~s}$ and $125 \mathrm{~s}$ for the quadratic and linear fits, respectively. The LTT effect of a third body in a circular orbit seen edge-on is

$$
\Delta T \simeq \frac{2 M_{3} G^{1 / 3}}{c}\left(\frac{P_{3}}{2 \pi\left(M_{1}+M_{2}\right)}\right)^{2 / 3}
$$

with $M_{1}, M_{2}, M_{3}$ being the masses of the three bodies and $P_{3}$ the period of the third body around the binary (Pribulla et al.2012). Taken the measured peak-to-peak amplitudes at face value, planetary masses of 22 and 38 Jupiter masses for an assumed period of 6.9 years are derived. Such masses are significantly larger than the typically reported (non-pathological) planets around 5 Jupiter masses of past studies but should be treated with great caution; the parameters are not well constrained by our study. As the above equation shows, the mass scales with the timing amplitude which is not really measured but estimated from the current peak-to-peak amplitude of the timing residuals.

The whole data set seems difficult to be reconciled with a single circumbinary planet due to the lack of regularity. On the other hand the data are not sufficient to finally constrain the parameters of a putative planetary system and to test the significance, the size, and physical interpretation of a quadratic term. This needs a much longer time base, in particular one needs to observe the turn-up towards later $(O-C)$ values.

A note on the timing errors might be in place here. The current fit results are dominated by the OPTIMA data points with timing uncertainties as small as $0.11 \mathrm{~s}$. This is smaller than the accretion area, hence at that precision a timing jitter due to accretion filaments or other forms of in-stationary accretion becomes relevant. Without a correction from the observed time of eclipse egress to eclipse center or without an extra error term which accounts for the finite size of the accretion area, searches for planets might be biased by the high precision of some of the data, which do not reflect the LTT effect of a circumbinary planet (planetary system) but the effects of in-stationary accretion.

The bottom line of this short communication is: The most recent addition to the $(O-C)$-data show an accelerated evolution of the timing residuals that rule out past models of a planet or a planetary system. HU Aqr deserves further monitoring with high cadence. Goździewski et al. (2012) have ignored data obtained at other than optical wavelengths to make their one-planet model applicable. The measurement presented here shows that this self-restriction doesn't save a one-planet model. It appears more advantageous to use the complete data set for future modeling. The current data were obtained with private equipment operated in a semi-professional manner. They were obtained with a comparatively small telescope (by professional standards) located at a mildly light-polluted site. Nevertheless a highly constraining data point could be determined. More advanced amateurs with similar equipment might become interested to join the enterprise of searching for long-period planets via the LTT effect in collaboration with professionals. Potential targets and work in this direction are described by Pribulla et al. (2012) and Backhaus et al. (2012).

Acknowledgements. We thank our referee for constructive criticism that help to improve the quality of the paper and Iris Traulsen and Robert Schwarz for help with the data reduction and useful discussions.

\section{References}

Applegate, J. H. 1992, ApJ , 385, 621

Backhaus, U., Bauer, S., Beuermann, K., et al. 2012, A\&A, 538, A84

Goździewski, K., Nasiroglu, I., Słowikowska, A., et al. 2012, MNRAS , 425, 930

Hakala, P. J., Watson, M. G., Vilhu, O., et al. 1993, MNRAS , 263, 61

Hinse, T. C., Lee, J. W., Goździewski, K., et al. 2012, MNRAS , 420, 3609

Horner, J., Marshall, J. P., Wittenmyer, R. A., \& Tinney, C. G. 2011, MNRAS , 416, L11

Portegies Zwart, S. 2013, MNRAS , 429, L45

Pribulla, T., Vaňko, M., Ammler-von Eiff, M., et al. 2012, Astronomische Nachrichten, 333, 754

Qian, S.-B., Liu, L., Liao, W.-P., et al. 2011, MNRAS , 414, L16

Schwarz, R., Schwope, A. D., Vogel, J., et al. 2009, A\&A, 496, 833

Schwope, A. D., Horne, K., Steeghs, D., \& Still, M. 2011, A\&A, 531, A34

Schwope, A. D., Mantel, K., \& Horne, K. 1997, A\&A, 319, 894

Schwope, A. D., Schwarz, R., Sirk, M., \& Howell, S. B. 2001, A\&A, 375, 419

Schwope, A. D., Thomas, H. C., \& Beuermann, K. 1993, A\&A, 271, L25 
Vogel, J., Schwope, A., Schwarz, R., et al. 2008, in American Institute of Physics Conference Series, Vol. 984, High Time Resolution Astrophysics: The Universe at Sub-Second Timescales, ed. D. Phelan, O. Ryan, \& A. Shearer, 264-267

Wittenmyer, R. A., Horner, J., Marshall, J. P., Butters, O. W., \& Tinney, C. G. 2012, MNRAS , 419, 3258 